\title{
Chlamydia pneumoniae and asthma
}

\author{
P J Cook, P Davies, W Tunnicliffe, J G Ayres, D Honeybourne, R Wise
}

Department of Respiratory Medicine, City Hospital, Dudley Road, Birmingham, UK

P J Cook

D Honeybourne

Department of

Respiratory Medicine, Birmingham

Heartlands Hospital,

Birmingham, UK

W Tunnicliffe

J G Ayres

Department of Microbiology, City Hospital, Dudley Road, Birmingham, UK

$\mathrm{R}$ Wise

School of Mathematics and Statistics, University of

Birmingham, Birmingham, UK

P Davies

Correspondence to: Dr P J Cook, Department of Respiratory Medicine, Birmingham Heartlands Hospital, Bordesley Green, Birmingham B9 5SS, UK.

Received 3 February 1997 Returned to authors 4 April 1997

Revised version received

1 December 1997

Accepted for publication

16 December 1997

\begin{abstract}
Background-This study was designed to test the association of Chlamydia pneumoniae infection with asthma in a multiracial population, after adjustments for several potential confounding variables. Methods-Antibodies to $C$ pneumoniae were measured by microimmunofluorescence in 123 patients with acute asthma, 1518 control subjects admitted to the same hospital with various non-cardiovascular, non-pulmonary disorders, and 46 patients with severe chronic asthma, including some with "brittle" asthma. Acute infection or reinfection was defined by titres of IgG of $\geqslant 512$ or IgM $\geqslant 8$ or a fourfold rise in IgG, and previous infection by IgG 64-256 or $\operatorname{IgA} \geqslant 8$. Logistic regression was used to control for likely confounders, including ethnic origin, age, sex, smoking habit, steroid medication, diabetes mellitus and social deprivation, on antibody levels.

Results-Antibody titres consistent with acute $C$ pneumoniae infection were found in $5.7 \%$ of patients with acute asthma and $5.7 \%$ of control patients, while $14.6 \%$ of patients with acute asthma and $12.7 \%$ of control patients had titres suggesting previous infection. These two groups did not differ significantly. However, titres suggesting previous infection were found in $34.8 \%$ of patients with severe chronic asthma: the difference between this group and the control group was statistically significant with an adjusted odds ratio of 3.99 (95\% confidence interval 1.60 to 9.97 ). Conclusions-These data raise important questions about the previously demonstrated association of $C$ pneumoniae infection with asthma, and suggest that future studies of this association should give particular attention to the presence or absence of a history of severe chronic asthma.

(Thorax 1998;53:254-259)
\end{abstract}

Keywords: Chlamydia pneumoniae; serology; asthma

Chlamydia pneumoniae, previously designated the TWAR agent, was first described in $1986 .{ }^{1}$ Serological studies indicate that it is one of the most prevalent infectious agents worldwide ${ }^{2-4}$ with a wide range of clinical manifestations including pneumonia, ${ }^{5}$ exacerbations of chronic obstructive pulmonary disease, ${ }^{6}$ and chronic asthma. ${ }^{7}$ There is also evidence that coronary and cerebral vascular disease are associated with serological evidence of this infection. $^{8}$

This study was designed to test the association of $C$ pneumoniae infection with asthma in a hospital serving the multiracial inner city population of north and west Birmingham, UK, adjusting for several potential confounding variables.

\section{Methods}

STUDY SUBJECTS AND INVESTIGATIONS

Approval for this project was obtained from the hospital ethical committees at the City and Heartlands Hospitals, Dudley Road, Birmingham. All subjects gave informed consent to participation.

Hospital admissions (acute asthma and controls) Patients with acute asthma and control subjects were admitted to Birmingham City Hospital via the Accident and Emergency Department. All patients were initially considered to be eligible; recruitment was prospective and continued at a steady rate so that those who were eventually included in the study were admitted consecutively throughout a 24 month period (March 1993 to March 1995). Exclusion criteria were known or suspected immunodeficiency, hypergammaglobulinaemia, connective tissue disease, and other autoimmune disease.

Patients with acute asthma were recruited on the basis of episodes of wheezing requiring immediate treatment with nebulised bronchodilators, on which all showed rapid clinical improvement. Further requirements for inclusion in this group were the absence of chronic bronchitis or emphysema (either from the patient's testimony or from the hospital case records) and the absence of a history of chronic sputum production. Where the responsible physician was in doubt about the diagnoses of asthma, the patient underwent formal measurements of forced expiratory volume in one second $\left(\mathrm{FEV}_{1}\right)$, forced vital capacity (FVC), and peak expiratory flow (PEF); the criteria for inclusion were then $\mathrm{FEV}_{1} / \mathrm{FVC}$ ratio $<0.7$ with an improvement of $\geqslant 15 \%$ in $\mathrm{FEV}_{1}, \mathrm{FVC}$, or $\mathrm{PEF}$ (or 0.2 litre in $\mathrm{FEV}_{1}$ alone) after nebulised $\beta_{2}$ agonist. Cases in which the diagnosis was uncertain, or which were complicated by active cardiovascular disease, were excluded from the study. Control subjects were selected randomly from all admissions to the Accident and Emergency Department with acute non-pulmonary, non-cardiovascular disorders, provided that there was no evidence of coexisting active cardiac, vascular, or pulmonary disease; thus there is no reason to suspect that they were predisposed to acquire $C$ pneumoniae infection.

Blood was taken from the patients with acute asthma and controls by the admitting physician on arrival in hospital (or within 12-24 hours where immediate venepuncture was impossible). All patients were asked to attend the out- 
Table 1 Demographic characteristics of acute asthma, chronic asthma, and control groups

\begin{tabular}{lllc}
\hline & $\begin{array}{l}\text { Acute asthma } \\
(n=123)\end{array}$ & $\begin{array}{l}\text { Chronic asthma } \\
(n=46)\end{array}$ & $\begin{array}{l}\text { Controls } \\
(n=1518)\end{array}$ \\
\hline $\begin{array}{l}\text { Ethnic origin } \\
\quad \text { Caucasian }\end{array}$ & $67(54.5 \%)$ & $45(97.8 \%)$ & $1061(69.9 \%)$ \\
$\quad$ Asian & $39(31.7 \%)$ & 0 & $290(19.1 \%)$ \\
$\quad$ Afro-Caribbean & $17(13.8 \%)$ & $1(2.2 \%)$ & $167(11.0 \%)$ \\
Age (years) & $16-82$ & $15-62$ & $16-88$ \\
$\quad$ Range & $45.5(16.2)$ & $36.2(11.4)$ & $56.5(18.0)$ \\
$\quad$ Mean (SD) & $47(38.2 \%)$ & $14(30.4 \%)$ & $844(55.6 \%)$ \\
Sex & $76(61.8 \%)$ & $32(69.6 \%)$ & $674(44.4 \%)$ \\
$\quad$ Male & $5.1(3.1)$ & $1.1(3.8)$ & $4.6(3.5)$ \\
$\quad$ Female & $27(22.0 \%)$ & $13(28.3 \%)$ & $448(29.5 \%)$ \\
Mean (SD) Townsend score & $12(9.8 \%)$ & $6(13.0 \%)$ & $264(17.4 \%)$ \\
Smoking habit & $82(66.6 \%)$ & $21(45.7 \%)$ & $689(45.4 \%)$ \\
$\quad$ Current & & & \\
$\quad$ Previous (>3 months) & $44(35.8 \%)$ & $43(93.5 \%)$ & $61(4.0 \%)$ \\
$\quad$ Never & $25(20.3 \%)$ & $3(6.5 \%)$ & $52(3.4 \%)$ \\
Steroid use & $53(43.1 \%)$ & 0 & $1393(91.8 \%)$ \\
$\quad$ Inhaled only & & & \\
$\quad$ Systemic & $1(0.8 \%)$ & NA & $54(3.6 \%)$ \\
$\quad$ None & $10(8.1 \%)$ & NA & $86(5.7 \%)$ \\
Diabetes mellitus & $10(8.1 \%)$ & NA & $58(3.8 \%)$ \\
$\quad$ Insulin dependent & & & \\
$\quad$ Non-insulin dependent & & &
\end{tabular}

$\mathrm{NA}=$ not available.
SEROLOGICAL TESTING

For each subject $2-5 \mathrm{ml}$ of serum was obtained by centrifugation within six hours and stored at $-20^{\circ} \mathrm{C}$ until analysis; testing of serum samples obtained on admission and during convalescence was deferred until both specimens were available. Each blood sample was labelled only with a serial number so the investigator was blind to all patient data at the time of testing and remained so until statistical analysis of the results.

Serum samples were tested by one investigator (PC) using Maxiscreen Chlamydia microimmunofluorescence (MIF) slides (IO International Ltd, London, UK) and FITC conjugated anti-human immunoglobulins. Only an even pattern of elementary body fluorescence was regarded as positive. In every batch of slides tested two control serum preparations known to be positive for this organism and two negative controls were each applied to two slides. All serum samples were screened at a dilution of $1: 8$; thereafter, positive samples were tested at dilutions of $1: 8$ to $1: 1024$.

Acute infection or reinfection just prior to entry into the study was presumed to be indicated by titres of $\operatorname{IgG} \geqslant 512, \operatorname{Ig} M \geqslant 8$ or, in patients who provided convalescent samples, IgG rising fourfold between initial and convalescent sampling. Titres indicating previous infection without recrudescence were presumed to be IgG $64-256$ or IgA $\geqslant 8$ provided that IgM could not be detected and there was no significant rise in IgG. Because patients with chronic asthma were not acutely ill when blood samples were taken, we did not expect to find evidence of acute $C$ pneumoniae (re)infection and were primarily interested in comparing frequencies of previous infection in this group.

Rheumatoid factor was assayed in patients in whom connective tissue diseases were suspected on clinical grounds despite insufficient evidence to exclude them from the study. $C$ pneumoniae $\operatorname{IgM}$ antibodies were discounted for serological classification where it was present because of its non-specific IgM reactivity. not meeting the criteria for diagnoses of "brittle" asthma). For type 1 "brittle" asthma these criteria were $\geqslant 40 \%$ daily variation in $\mathrm{PEF}$ (measured by the patient) for $\geqslant 50 \%$ of the time over a period of at least 150 days on high dose inhaled steroids. For type 2 "brittle" asthma the criteria were two or more life threatening exacerbations of otherwise stable asthma resulting in loss of consciousness, or type II respiratory failure with onset over less than 30 minutes and recovery within four hours of admission to hospital, despite intensive bronchodilator and steroid therapy. Blood samples were taken at routine outpatient appointments. Subjects were recruited to this part of the study during a 30 month period (March 1991 to September 1993).

Information on the presence of diabetes mellitus was not available for patients with chronic asthma. This potential confounding variable was therefore not included in the logistic regression analysis.

\section{ANALYSIS OF DATA}

The raw frequencies of acute (re)infection and previous infection were compared in patients with acute asthma, chronic asthma, and controls by simple univariate analyses using $\chi^{2}$ tests. A logistic regression modelling method, as implemented in the EGRET statistical package, was used to explore possible influences of broad ethnic origin (Caucasian, Asian or Afro-Caribbean), age ( \pm 10 years), sex, smoking habit (current or previous versus never), steroid use, and social deprivation on any association of antibody levels with asthma. Hence we derived odds ratios expressing the associations of acute asthma with acute $C$ pneumoniae (re)infection and previous infection and of chronic asthma with previous infection, adjusted for potential confounding factors. 
Table 2 IgG titres and serological classification of acute asthma, chronic asthma, and control groups

\begin{tabular}{llll}
\hline & Acute asthma (n=123) & Chronic asthma (n=46) & Controls (n=1518) \\
\hline IgG titres & & & $0-512$ \\
Range & $0-512$ & $0-256$ & 0 \\
Median & 0 & 16 & 19.6 \\
Mean & 24.3 & 33.7 & $87(5.7 \%)$ \\
Acute (re)infection & $7(5.7 \%)$ & $2(4.3 \%)$ & 1.00 \\
$\quad$ Unadjusted odds ratio (95\% CI) & $1.02(0.46$ to 2.25$)$ & $1.02(0.24$ to 4.34$)$ & 1.00 \\
Adjusted odds ratio (95\% CI) & $0.81(0.32$ to 2.05$)$ & $1.07(0.13$ to 8.65$)$ & $1.03(12.7 \%)$ \\
Previous infection & $18(14.6 \%)$ & $16(34.8 \%)$ & 1.00 \\
$\quad$ Unadjusted odds ratio (95\% CI) & $1.18(0.70$ to 1.99$)$ & $3.66(1.95$ to 6.90$)$ & $1238(81.6 \%)$ \\
Adjusted odds ratio $(95 \% \mathrm{CI})$ & $0.92(0.49$ to 1.70$)$ & $3.99(1.60$ to 9.97$)$ & \\
No infection & $98(79.7 \%)$ & $28(60.9 \%)$ & \\
\hline
\end{tabular}

\section{Results}

STUDY SUBJECTS AND INVESTIGATIONS

Twenty seven patients with acute asthma and 312 potential controls were excluded from the study by the criteria stated above, and seven and 53 subjects, respectively, refused to participate. A total of 1687 patients were recruited and their demographic characteristics are shown in table $1 ; 123(7.3 \%)$ had been admitted with acute asthma, in four of whom the responsible physicians were uncertain about the diagnosis and referred them for formal pulmonary function tests which confirmed the presence of asthma by the criteria stated above. Forty six $(2.7 \%)$ were assigned to the chronic asthma group; 17 (four men) had type 1 "brittle" asthma, 11 (four men) had type 2 "brittle" asthma, and 18 (four men) were stable severe chronic asthmatics. Many of these patients had important co-morbidities including diabetes mellitus.

The remaining 1518 patients $(90.0 \%)$ were assigned to the control group. In these patients the primary indications for admission to hospital were abdominal pain $(n=460)$ for which causes were established in 403 cases; chest pain not suggestive of myocardial ischaemia and for which no cause was found $(\mathrm{n}=91)$; malignant disease $(n=85)$; fractures of various bones ( $n$ $=77$ ); urinary obstruction $(n=64)$; complications of diabetes mellitus $(n=44)$; and other miscellaneous medical and surgical diagnoses ( $\mathrm{n}=697$ ).

Approximately $4 \%$ of control patients were taking inhaled steroids at the time of admission to hospital. However, these patients had no evidence of active pulmonary disease and therefore lacked standard indications for inhaled steroids. Control patients who were taking systemic steroids all did so for nonpulmonary, non-cardiovascular diseases.

Twenty nine patients with acute asthma and 282 control patients $(23.6 \%$ and $18.5 \%$, respectively) attended for convalescent blood sampling within 160 days at a mean (SD) of 98.2 (37.6) days following admission. After 160 days the serum samples were no longer considered to be convalescent samples and they were discarded.

All patients known to have connective tissue or other autoimmune disease were excluded, after which there remained in the study 13 patients with acute asthma and 133 controls in whom connective tissue diseases were suspected on clinical grounds and who were therefore tested for the presence of rheumatoid factor. Where this test was positive (in four patients with acute asthma and 24 controls) the finding of $\operatorname{IgM}$ antibodies was discounted for the diagnosis of acute $C$ pneumoniae infection.

Acute infection was diagnosed solely on the basis of $\operatorname{IgM}$ titres in two patients with acute asthma and 30 controls, of whom both of the patients with acute asthma and 22 of the controls would otherwise have been defined by their IgG titres as previously infected. Eleven of the controls, but none of the patients with acute asthma, were older than 60 years and therefore were within the age group that has been associated with unsuspected rheumatoid factor production. ${ }^{9}$

\section{PNEUMONIAE AND ACUTE ASTHMA}

Immunoglobulin titres and serological classifications are shown in table 2 . On $\chi^{2}$ analysis there was no significant difference in the distributions of the three possible serological outcomes (acute (re)infection, previous infection, and no infection) between the acute asthma and control groups. The odds ratios for acute and previous $C$ pneumoniae infection in patients with acute asthma compared with control subjects, unadjusted and adjusted by logistic regression for ethnic origin, sex, smoking habit, age and Townsend score, are shown in table 2 with $95 \%$ confidence intervals (CI). There was no evidence of an interaction between these potential confounding factors and acute asthma or $C$ pneumoniae infection.

Ignoring IgM antibody titres from the 11 control patients over 60 years of age, not tested for rheumatoid factor, in whom we detected acute $C$ pneumoniae infection solely on the basis of $\operatorname{IgM}$ would have made no difference to this lack of association between acute asthma and either acute $C$ pneumoniae (re)infection or previous infection.

C PNEUMONIAE AND SEVERE CHRONIC ASTHMA The results from this group are also shown in table 2. Two of the patients with type 2 "brittle" asthma appear to have had acute (re)infection at or shortly before the time of sampling. On $\chi^{2}$ analysis the distributions of the three possible serological outcomes (acute (re)infection, previous infection, and no infection) differed significantly between the patients with chronic asthma and controls $(\mathrm{p}<0.05)$, this difference being attributable to previous infection. There was no evidence of an interaction between the potential confounding factors and chronic asthma or $C$ pneumoniae infection. 
These numbers are too small to permit a rigorous comparison of the subgroups of chronic severe asthma.

\section{Discussion}

C PNEUMONIAE AND ASTHMA

Asthma is a chronic inflammatory disorder ${ }^{10}$ in which eosinophils appear to play a major part, irrespective of the presence or absence of an atopic diathesis, ${ }^{11}$ but the relevance of pulmonary infection to this condition remains uncertain. ${ }^{12}$ Chronic infection with $C$ pneumoniae has been documented ${ }^{13}$ and, since chlamydial lung infections commonly cause wheezing,${ }^{14}$ it is plausible that chronic infection would produce a tendency to chronic asthma.

In a study of 365 adults with acute respiratory illness acute $C$ pneumoniae infection was diagnosed serologically in 19 patients $(5.2 \%)$, of whom nine $(47.3 \%)$ reported wheezing. ${ }^{7}$ A polyvalent $C$ pneumoniae antibody titre of $\geqslant 64$ conferred a high risk of wheezing, and there was a clear dose-response relationship between antibody titre and the presence of wheezing. Later diagnoses of asthmatic bronchitis were made within six months of the presenting illness in $29.6 \%$ of patients with antibody titres of $\geqslant 64$ at recruitment, and in only $7 \%$ of matched controls $(\mathrm{p}<0.05)$. Antibodies to this organism were more likely to be found in adults with late onset asthma but no atopy (as judged by negative skin tests) than in those who were atopic or whose asthma started in childhood. ${ }^{15}$

Several other groups have also shown a serological association with this organism in chronic adult asthma. ${ }^{16}{ }^{17}$ Furthermore, population based longitudinal data from Helsinki suggest that increasing $C$ pneumoniae seroprevalence is associated with increasing incidence of asthma attacks in all age groups. ${ }^{18}$

Our data support an association of previous $C$ pneumoniae infection with severe chronic asthma in the absence of exacerbations. The lack of outpatient controls might be criticised, but the comparison with inpatients (for previous infection only) can be justified on the grounds that blood samples were taken from them within 24 hours of admission to hospital and there was therefore insufficient time to acquire any infections nosocomially. However, the number of patients with chronic asthma recruited to this study was small and further investigation is therefore warranted.

Our results do not support an association between $C$ pneumoniae antibody titres and the incidence of acute asthma attacks. This is in agreement with a small study by Simon et al. ${ }^{19}$ The apparent conflict with other studies $^{716}$ might be due to the small numbers of patients returning for convalescent blood sampling, or to the fact that the power of our study to detect such an association was limited by the relatively small number of subjects with acute asthma. However, in other studies it is not clear how many subjects had chronic asthma. In our study the 123 patients recruited to the acute asthma group included only 13 with documented reversible airflow obstruction prior to the onset of the presenting illness. It is possible that an association between $C$ pneumoniae infection and asthma was merely masked by the heterogeneity of this group. Furthermore, asthma is not a homogeneous condition and exacerbations may be provoked by a wide range of infectious and non-infectious factors. Any effect of $C$ pneumoniae infection in promoting the development of asthma could therefore have been obscured by other factors, at least during the period of the study.

Strategies to take account of potential confounding variables are essential in studies of $C$ pneumoniae antibodies, which have been associated with Afro-Caribbean origin, ${ }^{20}$ the use of steroid medication, ${ }^{21}$ increasing age, ${ }^{22}$ and male sex. ${ }^{22}$

Few previous studies of $C$ pneumoniae infection have addressed the smoking habits of the subjects. ${ }^{23}$ However, smokers have in general a higher risk of respiratory infection than non-smokers. ${ }^{24}{ }^{25}$ Evidence that they may also be more susceptible to $C$ pneumoniae infection was published in $1992 .{ }^{26}{ }^{27} \mathrm{~A}$ greater propensity among smokers to acquire $C$ pneumoniae infection might create a spurious association between this infection and pulmonary disease. This hypothesis is supported by a reported association between smoking and seroconversion to $C$ pneumoniae as detected by microimmunofluorescence. ${ }^{26} 28$

Socioeconomic deprivation must also be considered as a potential confounding variable. In the UK social deprivation is clearly correlated with overall morbidity ${ }^{29}$ but not with asthma or chronic bronchitis. (Obstructive lung diseases may more readily be labelled "bronchitis" than "asthma" when they afflict people from lower socioeconomic groups.) Asthma is rare in developing countries ${ }^{30} 31$ and less common in rural than in urban districts, so it might be considered a disease of affluence in such countries. In developed countries, where urban dwelling has no clear association with income, asthma appears to have a higher prevalence in towns. These observations suggest that environmental factors are more important than poverty.

Townsend scores, which include items that reflect predominantly personal and family income, may be considered to reflect the socioeconomic status of people living within a particular locality. ${ }^{32}$ They include items that reflect predominantly personal and family income and were therefore considered to be superior to the Jarman score (developed as a predictor of the demand for primary medical care) for an assessment of the effects of living standards on health. In our study Townsend scores showed no correlation with asthma or with serological evidence of $C$ pneumoniae infection.

By logistic regression analysis we found no noteworthy statistical interactions-that is, effect modifications in different subgroups - of ethnic origin, age, sex, steroid use, smoking habit, or Townsend score with the $C$ pneumoniae/asthma associations. Furthermore, where data on diabetes mellitus were available - that is, in the acute asthma and 
control groups-we detected no interaction with diabetes.

SEROLOGICAL TESTING

Various techniques are available to detect $C$ pneumoniae antibodies. ${ }^{33}$ The best and most widely used is the MIF assay which is sensitive and species-specific and reliably detects IgG, $\operatorname{IgM}$, and $\operatorname{IgA} .{ }^{34}$ The kit which we used has been employed in several studies. ${ }^{235}$

Because only a small proportion of patients in each group gave convalescent serum samples within 160 days, the acute (re)infection rate ascertained by a fourfold rise in IgG should be considered to represent a minimum for each group. In a single serum specimen an IgG titre of $\geqslant 512$ can also be interpreted as evidence of acute primary infection ${ }^{36}$ or, particularly in older subjects, reinfection or recrudescence of chronic or latent infection. ${ }^{37}$ IgG titres of $\leqslant 256$ have generally been accepted (in a single serum specimen) as evidence of previous infection ${ }^{6}$ provided that there are no $\operatorname{Ig} M$ antibodies. Many authors have employed these serological criteria $^{38-45}$ and a large study combining serological data with an examination of pharyngeal swabs by polymerase chain reaction has provided evidence in support of them. ${ }^{46}$ Our decision to include IgA is justified by evidence that it persists for more than three weeks in chronic infection. ${ }^{47} 48$

$\operatorname{IgM}$ is generally considered to signify acute primary infection. ${ }^{49} \mathrm{~A}$ threshold titre of $\geqslant 16$ has been proposed with titres of 8 indicating "probable acute infection", ${ }^{40}$ but we found no difference in the proportions of patients with IgM antibodies at these two titres in a large pilot study and therefore saw no merit in drawing a distinction between such low levels of antibody production. It has been suggested ${ }^{50}$ that rheumatoid factor may make the measurement of $\operatorname{IgM}$ antibodies unreliable, particularly in elderly patients. ${ }^{9}$ We therefore excluded from this study all patients known to have connective tissue or other autoimmune diseases and measured rheumatoid factor in a further 146 cases in which such diseases were considered less probable, discounting IgM antibodies in the 28 cases in which it was present. As shown above, ignoring IgM titres from the 11 remaining patients over 60 years of age would not have significantly altered the relationships of acute and chronic asthma to acute and chronic $C$ pneumoniae infections.

We conclude that these data do not support an association between the seroprevalence of $C$ pneumoniae in the general population and acute asthma, but they do suggest that there may be an association with severe chronic asthma. In future studies researchers should specify whether subjects were previously asthmatic (particularly whether they had "brittle" or other severe chronic asthma), and their ethnic origin and medication.

At the City Hospital, Birmingham we wish to acknowledge the helpful advice of Mr Richard Matthews (Virology Laboratory), Melpful advice of Mr Richard Matthews (Virology Laboratory), Mebron and Mr Peter Cooke (Pharmacy). At the University of Birmingham epidemiological and statistical assistance was given by Dr Sarah O'Brien, Dr Caren Grainger, and other staff of the Department of Public Health Epidemiology. At the University of London the advice and support of Dr John Treharne (Reader in Virology) and Professor S Darougar (Emeritus Professor of Public Health Ophthalmology) are also gratefully acknowledged. Ms Sue Dobson, Ms Gill Curley and Ms Helen Kellaway (City Hospital) assisted in locating hospital case notes and $\mathrm{Mr}$ Derek Price (Bionostics Ltd) and Ms Maria van Kampen kindly assisted in the labelling and sorting of blood specimens. We wish to thank IO International Ltd, London for their generous support of this project. PJC has received financial assistance from the Peel Medical Research Trust and Allen \& Hanburys. We also thank the referees of earlier drafts of this paper for their helpful comments and suggestions.

1 Grayston JT, Kuo CC, Wang SP, et al. A new Chlamydia psittaci strain, TWAR, isolated in acute respiratory tract psittaci strain, TWAR, isolated in acute

2 Forsey T, Darougar S, Treharne JD, et al. Prevalence in human beings of antibodies to Chlamydia IOL-207, an atypical strain of Chlamydia. F Infect 1986;12:145-52.

3 Kanamoto Y, Ouchi K, Mizui M, et al. Prevalence of antibody to Chlamydia pneumoniae TWAR in Japan. $\mathcal{F}$ Clin Microbiol 1991;29:816-8.

4 Montes M, Cilla G, Alcorta M, et al. High prevalence of Chlamydia pneumoniae infection in children and young adults in Spain. Pediatr Infect Dis f 1992;11:972-3.

5 Saikku P, Wang SP, Kleemola M, et al. An epidemic of mild pneumonia due to an unusual strain of Chlamydia psittaci. $\mathcal{F}$ Infect Dis 1985;151:832-9.

6 Beaty CD, Grayston JT, Wang SP, et al. Chlamydia pneumoniae, strain TWAR, infection in patients with chronic obstructive pulmonary disease. Am Rev Respir Dis 1991; 144:1408-10.

7 Hahn DL, Dodge R, Golubjatnikov R. Association of Chlamydia pneumoniae (strain TWAR) infection with wheezing, asthmatic bronchitis and adult-onset asthma. AMA 1991;266:225-30.

8 Cook PJ, Honeybourne D. Clinical aspects of Chlamydia pneumoniae infection. Presse Med 1995;24:278-82.

9 Verkooyen RP, Hazenberg MA, van Haaren GH, et al. Agerelated interference with Chlamydia pneumoniae microimmunofluorescence serology due to circulating rheumatoid factor. F Clin Microbiol 1992;30:1287-90.

10 National Asthma Education Program. Guidelines for the diagnosis and management of asthma. F Allergy Clin Immunol 1991;88(Suppl):425-534.

11 Frigas E, Gleich GJ. The eosinophil and the pathophysiology of asthma. F Allergy Clin Immunol 1986;77:527-37.

12 Stenius-Aarniala B. The role of infection in asthma. Chest 1987;91:130-6S.

13 Yamazaki T, Nakada H, Sakurai N, et al. Chlamydia pneumoniae, strain TWAR, infections of young children in a Japanese family. In: Bowie WR, Caldwell HD, Jones RP, et al, eds. Chlamydial infections. Cambridge: Cambride University Press, 1990: 433-6.

14 Frydén A, Kihlström E, Maller R, et al. A clinical and epidemiological study of "ornithosis" caused by Chlamydia psittaci and Chlamydia pneumoniae (strain TWAR). Scand $\mathcal{F}$ Infect Dis 1989;21:681-91.

15 Hahn DL, Golubjatnikov R. Age at asthma diagnosis, skin test positivity and Chlamydia pneumoniae seroreactivity. Am 7 Respir Crit Care Med 1994;149:913A.

16 Allegra L, Blasi F, Centanni S, et al. Acute exacerbations of asthma in adults: role of Chlamydia pneumoniae infection. Eur Respir $\mathcal{F}$ 1994; 7:2165-8.

17 Peters BS, Thomas B, Marshall B, et al. The role of Chlamydia pneumoniae in acute exacerbations of asthma. $\mathrm{Am} \mathcal{F}$ Respir Crit Care Med 1994;149:34A.

18 Puolakkainen M, Ukkonen P, Saikku P. The seroepidemiology of Chlamydiae in Finland over the period 1971 to 1987. Epidemiol Infect 1989;102:287-95.

19 Simon FT, Nelson HS, Noda JA, et al. The role of Chlamydia pneumoniae in reversible obstructive airway disease. $\mathcal{F}$ Allergy Clin Immunol 1996;97:306.

20 Cook PJ, Honeybourne D, Wise R, et al. Chlamydia pneumoniae antibody titres are significantly associated with Afro-Caribbean and Asian origin. Eur Respir f 1996; 9(Suppl 23):326s

21 Cook PJ, Davies P, Honeybourne D, et al. Chlamydia pneumoniae antibody titres are significantly associated with the use of steroid medication in respiratory disease. Eur Respir f 1996;9(Suppl 23):5s.

22 Paltiel O, Kark JD, Leinonen M, et al. High prevalence of antibodies to Chlamydia pneumoniae: determinants of IgG and IgA seropositivity among Jerusalem residents. Epidemiol Infect 1995;114:465-73.

23 Van den Broucke JP, Kosenvuo M, Romanov K. Chlamydia TWAR and acute myocardial infarction. Lancet 1989;i:158.

24 Blake GH, Abell TD, Stanley WG. Cigarette smoking and upper respiratory infection among recruits in basic combat training. Ann Intern Med 1988;109:198-202.

25 Mili F, Flanders WD, Boring JR, et al. The associations of race, cigarette smoking and smoking cessation to measures of the immune system in middle-aged men. Clin Immunol Immunopathol 1991;59:187-200.

26 Hahn DL, Golubjatnikov R. Smoking is a potential confounder of the Chlamydia pneumoniae-coronary artery
disease association. Arteriosclerosis Thromb 1992;12:945-7.

27 Saikku P, Leinonen M, Tenkanen L, et al. Chronic Chlamydia pneumoniae infection as a risk factor for coronary heart
disease in the Helsinki heart study. Ann Intern Med 1992;116:273-8.

28 Saikku P. The epidemiology and significance of Chlamydia pneumoniae. F Infect 1992;25(Suppl 1):27-34 
29 Department of Health and Social Security. Inequalities in health: report of a Research Working Group. London: HMSO

30 Godfrey RC. Asthma and IgE levels in rural and urban communities of the Gambia. Clin Allergy 1975;5:201-7.

31 Van Niekerk CH, Weinberg EG, Shore SC, et al. Prevalence of asthma: a comparative study of urban and rural Xhosa children. Clin Allergy 1979;9:319-24.

32 Townsend P, Phillimore P, Beattie A. Health and deprivation. London: Routledge, 1989.

33 Cook PJ, Honeybourne D. Chlamydia pneumoniae. 7 Antimicrob Chemother 1994;34:859-73.

34 Csángó PA, Sarov B, Schiotz H, et al. Comparison between cell culture and serology for detecting Chlamydia trachomatis in women seeking abortion. F Clin Pathol 1988;41:8992 .

35 Moss TR, Darougar S, Woodland RM, et al. Antibodies to Chlamydia species in patients attending a genitourinary clinic and the impact of antibodies to $C$ pneumoniae and $C$ psittaci on the sensitivity and the specificity of $C$ trachomatis psittaci on the sensitivity and the specificity of
serology tests. Sex Transm Dis 1993;20:61-5.

36 Grayston JT, Campbell LA, Kuo CC, et al. A new respiratory tract pathogen: Chlamydia pneumoniae, strain TWAR. F Infect Dis 1990;161:618-25.

37 Aldous MB, Grayston JT, Wang SP, et al. Sero-epidemiology of Chlamydia pneumoniae TWAR infection in Seattle families, 1966-1979. F Infect Dis 1992;166:646-9.

38 Saikku P, Ruutu P, Leinonen M, et al. Acute lower respiratory tract infection associated with chlamydial TWAR antibody in Filipino children. F Infect Dis 1988;158:1095-7.

39 Grayston JT, Mordhorst CH, Bruu AL, et al. Country-wide epidemics of Chlamydia pneumoniae, strain TWAR, in Scandinavia, 1981-1983. F Infect Dis 1989;159:1111-4.

40 Mordhorst CH, Wang SP, Myhra W, et al. Chlamydia pneumoniae, strain TWAR, infections in Denmark, 1975-1987.
In: Bowie WR, Caldwell HD, Jones RP, et al, eds. Chlamydial infections. Cambridge: Cambridge University Press, 1990: 418-21.

41 Berdal BP, Scheel O, Ygaard AR, et al. Spread of subclinical Chlamydia pneumoniae infection in a closed community. Scand $\mathcal{F}$ Infect Dis 1992;24:431-6.

42 Ozanne G, Lefebvre J. Specificity of the microimmunofluorescence assay for the serodiagnosis of Chlamydia pneumoniae infections. Can 7 Microbiol 1992;38:1185-9.

43 Ekman MR, Grayston JT, Visakorpi R, et al. An epidemic of infections due to Chlamydia pneumoniae in military infections due to Chlamydia pneumoniae
conscripts. Clin Infect Dis 1993;17:420-5.

44 Freidank HM, Brauer D. Prevalence of antibodies to Freidank HM, Brauer D. Prevalence of antibodies to
Chlamydia pneumoniae TWAR in a group of German medical students. F Infect 1993;27:89-93.

45 Kern DG, Neill MA, Schachter J. A seroepidemiologic study of Chlamydia pneumoniae in Rhode Island: evidence of serologic cross-reactivity. Chest 1993;104:208-13.

46 Thom DH, Grayston JT, Campbell LA, et al. Respiratory infection with Chlamydia pneumoniae in middle-aged and older adult outpatients. Eur F Clin Microbiol Infect Dis 1994; 13:785-92.

47 Sarov I, Sarov B, Hanuka N, et al. The significance of serum specific IgA antibodies in diagnosis of active Chlamydia trachomatis infections. In: Oriel JD, Ridgway GL, Schachter J, eds. Chlamydial infections. Cambridge: Cambridge University Press, 1986: 566-9.

48 Samra Z, Soffer Y. IgA anti-Chlamydia antibodies as a diagnostic tool for monitoring of active chlamydial infection. Eur F Epidemiol 1993;8:882-4.

49 Wang SP, Grayston JT. Human serology in Chlamydia trachomatis infection with micro-immunofluorescence. F Inf Dis 1974;130:388-97.

50 Marrie TJ. Chlamydia pneumoniae. Thorax 1993;48:1-4. 